\title{
Linx
}

Revue des linguistes de l'université Paris X Nanterre

9 | 1997

Émile Benveniste. Vingt ans après

\section{Benveniste et le paradigme de l'énonciation}

\section{Sungdo Kim}

\section{OpenEdition \\ Journals}

Édition électronique

URL : http://journals.openedition.org/linx/1051

DOI : 10.4000/linx.1051

ISSN : 2118-9692

\section{Éditeur}

Presses universitaires de Paris Nanterre

\section{Édition imprimée}

Date de publication : 1 avril 1997

Pagination : 211-218

ISSN : 0246-8743

\section{Référence électronique}

Sungdo Kim, « Benveniste et le paradigme de l'énonciation », Linx [En ligne], 9 | 1997, mis en ligne le 06 juillet 2012, consulté le 30 avril 2019. URL : http://journals.openedition.org/linx/1051 ; DOI : 10.4000/ linx.1051 


\title{
Benveniste et le paradigme de l'énonciation
}

\author{
Sungdo Kim
}

\section{$P_{\text {réliminaires }}$}

L'Oeuvre de Benveniste me parait avant tout une belle synthèse de la théorie du langage et de la recherche empirique des langues. Un coup d'oeil sur sa référence majeure "Problèmes de linguistique générale" abrégé dorénavant PLG permet de révéler la profondeur et l'étendue de ses domaines de recherche. Il cite une centaine de savants de son temps et du passé et traite 114 langues. Evidemment l'originalité de son oeuvre n'est pas là. Sa contribution fondamentale consistait à précipiter la fin de l'immanentisme en prenant pour position ontologique radicale d'envisager le réel du langage et de l'insérer dans l'appareil théorique. Les arguments de cette communication peuvent se résumer ainsi : sur le plan de l'ontologie du langage l'apport de Benveniste est éclairant en rénovant le cadre structurel par le recours à deux "bêtes noires" de la linguistique d'inspiration saussurienne: le référent et le sujet. Autrement dit, la réalité qui est évacuée du noyau structural est revenue comme un fantôme dans la maison de Benveniste. Ce geste, je l'appellerai pour ma part, le réalisme benvenistien. Mais sur le plan de l'épistémologie c'est-àdire sur le plan de la théorisation linguistique, la théorie de l'énonciation de Benveniste est insuffisante sinon incomplète pour recevoir le statut de théorie, malgré ses idées brillantes et la prolifération des éloges qu'elle a reçus.

Avant d'approfondir ces arguments, je me permet d'esquisser de façon négative le profil de cet exposé. Il ne s'agit pas de la réception de ses idées dans le milieu structuraliste (cf. Normand 1985), ni de l'enchâssement historique de son paradigme énonciatif (cf. Delesalle 1986). Elle ne porte pas non plus sur l'actualité du Benvenistisme. Enfin le surgissement de ce concept ne sera pas évoqué ici. 
Sungdo Kim

Je me limite résolument à la perspective épistémologico-historique, à savoir que je vise à construire une problématique sur la structure et les sources de sa théorie de l'énonciation. En même temps, je tenterai d'exposer mes doutes avec brièveté sur les fondements épistémologiques.

1. D'abord j'aimerais dire quelques mots sur la structure interne de la théorie de Benveniste. Déjà dans ses travaux indo-européens (cf. Coquet 1987), le jeune Benveniste a conçu les rudiments de la théorie de l'énonciation dans l'analyse morphologique des noms d'agent et elle s'est développée pendant 20 ans. L'essentiel de ce paradigme se condense dans l'article qui, publié en 1970, s'intitule "L'appareil formel de l'énonciation". Il y réfute le principe formalisant $\mathrm{du}$ langage en mettant l'accent sur l'action du langage. Il s'agit d'une conception réaliste de la langue. Dans ce cadre Benveniste fait attention d'abord à l'emploi des formes dont les conditions ne sont pas identiques à celles de la langue. C'est ici que sa fameuse définition de l'énonciation se présente. Voici deux passages à ce propos :

"L'énonciation est cette mise en fonctionnement de la langue par un acte individuel d'utilisation" (PLG 2, 80).

"Le discours, dira-t-on, qui est produit chaque fois qu'on parle, cette manifestation de l'énonciation, n'est pas simplement la «parole»(... ) C'est l'acte même de produire, un énoncé et non le texte de l'énoncé qui est notre objet. Cet acte est le fait du locuteur qui mobilise la langue pour son compte" (ibid.).

Benveniste mentionne trois aspects principaux participant à ce processus de la production énonciative :la réalisation vocale, la conversion individuelle de la langue en discours, la sémantisation de la langue. Si je transpose ces trois éléments en d'autres termes ce sera : 1 . la présence du matériel signifiant 2 . la participation des utilisateurs du langage 3 . la réalité extra-linguistique à quoi se réfèrent les séquences de signes produites en contexte. Ainsi, il exploite une voie qui permet de définir l'énonciation dans le cadre formel de sa réalisation en faisant recours à l'acte et à la situation où cet acte se réalise. La théorie de l'énonciation présuppose par conséquent l'appropriation, c'est-à-dire la subjectivation, et en même temps l'interlocuteur, et finalement envisage le monde.

Plus précisément nous pouvons récupérer quatre instances qui participent à la configuration de la théorie de l'énonciation. En premier lieu, il s'agit de la subjectivité dans le langage. Le commentaire est inutile à ce propos dans la mesure où Benveniste la définit mieux que personne :

"Le locuteur s'approprie l'appareil formel de la langue et il énonce sa position du locuteur par des indices spécifiques" (Ibid., 82).

"La «subjectivité» dont nous traitons ici est la capacité du locuteur à se poser comme «sujet». Elle se définit, non par le sentiment que chacun éprouve d'être lui-même (... ) 
mais comme l'unité psychique qui transcende la totalité des expériences vécues qu'elle assemble et qui assure la permanence de la conscience" (PLG 1, 259-260).

Cela signifie l'expérience subjective des sujets parlants qui se posent et situent dans le langage et à travers lui. La dialectique de je et tu appartient à cette subjectivité. Déjà à partir des années 1950, Benveniste fait la triple référence : personne, nombre, diathèse pour saisir le sujet dans le processus. Ceci permet de définir ce que Benveniste appelle "le champ positionnel du sujet" (PLG 1, 174).

En deuxième lieu, il s'agit du dialogisme ou de l'intersubjectivité. En effet toute énonciation est une allocution; elle postule un allocutaire. Ce qui suppose l'intersubjectivité de l'énonciation. Il est vrai que, tandis que Benveniste focalise sur le sujet énonciateur, la place de l'intersubjectivité reste mineure. Pourtant cela n'empêche pas qu'une certaine conception de l'altérité laisse quelques traces. Car le régime dialogique et pragmatique de l'altérité est inéliminable à partir du moment où les différences se maintiennent en situation référentielle effective. Selon la terminologie de Francis Jacques, il s'agit de l'altérité de relation qui n'est pas sans pouvoir et qui invente la nouvelle problématique. (cf. Jacques 1982, 1985).

En troisième lieu, la référence est fondamentale, c'est-à-dire que dans l'énonciation "la langue se trouve employée à l'expression d'un certain rapport au monde" (Ibid., 82). En d'autres termes, avec la fonction référentielle qui désigne les êtres ou décrit les états de choses, le langage tente de coller à la réalité ou adhérer aux choses :

"La condition de cette mobilisation et de cette appropriation de la langue est, chez le locuteur, le besoin de référer par le discours, et chez l'autre, la possibilité de co-référer identiquement, dans le consensus pragmatique qui fait de chaque locuteur un co-locuteur. La référence est partie intégrante de l'énonciation" (Ibid., 82).

Quatrièmement, la prédication est indispensable pour l'efficacité sémantique. Dans ce cadre les énonciativistes s'opposent nettement aux logiciens. Déjà Sechehaye affirme que la logique n'est pas dans les choses, elle est en nous dans ce que nous pensons à propos des choses (cf. 1926). En effet l'énoncé construit et décrit les nouvelles représentations en modifiant les représentations mentales. Dans le cadre de la prédication Benveniste définit le temps linguistique comme l'opération qui repère le temps prédiqué par rapport au temps de la situation d'énonciation. Il s'agit de deux plans d'énonciation. Le discours, où le fait est présenté comme lié à l'énonciateur, et l'histoire, où le fait est présenté sans intervention de l'énonciation.

Bref, l'opération prédicative exige les interlocuteurs. Par exemple, une assertion n'est pas vraie en soi ; elle l'est du point de vue des interlocuteurs qui 
Sungdo Kim

l'évaluent . Autrement dit, la prédication n'existe que sous la dépendance d'un certain acte d'énonciation. Voici ce que dit Coquet à propos de l'importance de la prédication :

"Par la prédication nous manifestons notre insertion dans le monde. Le second domaine de réalité, que l'on peut considérer comme le corrélat objectif du premier, est celui de l'assertion. Il n'y a pas de sujet qui soit privé de prédication" (Coquet, 1991, 33)

2. Dans la deuxième partie de cet exposé j'aimerais dire quelques mots sur les sources historico-épistémologiques de la théorie de l'énonciation. On peut déceler les trois gestes majeurs qui auraient inspiré l'élaboration de son appareil dit énonciatif: ce sont la sémiologie, la pragmatique et la phénoménologie. Mais aucune de ces sources n'a été reçue dans son ensemble ; l'appréhension de Benveniste vis-à-vis de ces trois versants se révèle partielle ou tronquée même.

En premier lieu, la sémiologie, ici, doit s'entendre dans une large conception indépendamment d'une école particulière. C'est l'équivalent de l'anthropologie de l'homme. En effet l'ambition anthropologique s'exprime chez Benveniste dans son programme de sémiologie ; Le caractère englobant du projet sémiologique s'affirme (cf. Normand 1989). Le projet s'impose en tant que théorie du sens et du symbolique en général :

"dans une certaine culture, comme dans une langue, il y a un ensemble de symboles dont il s'agit de définir les relations... C'est du progrès de l'analyse des symboles qu'on pourrait atteindre notamment une meilleure compréhension..." (PLG 1, 11-12).

A cet égard, l'article promulgateur de Benveniste "Sémiologie de la langue" est à relire. En ce qui concerne la lecture de Peirce, signalons au passage que, demeurant foncièrement dualisant et saussurien, Benveniste échoue à pénétrer dans les idées originelles du sémioticien pragmatique. Les deux mots qui caractérisent l'attitude de Benveniste sont à la fois fascination et panique.

"Peirce a posé une triple division des signes, en ICÔNES, INDEX et SYMBOLES qui est à peu près tout ce qu'on retient aujourd'hui de l'immense architecture logique qu'elle sous-tend" (PLG 11, 44).

et tout de suite la panique se manifeste :

"En ce qui concerne la langue, Peirce ne formule rien de précis ni de spécifique. Pour lui la logique est partout et nulle part. Il ne s'est jamais intéressé au fonctionnement de la langue, si même il y a prêté attention..."(ibid.).

Benveniste renie Peirce qui avait dit des choses qu'il va répéter du fonctionnement et des modalités du signe. La typologie que Benveniste nous en propose n'est qu'une partie infime du projet peircien. 
En deuxième lieu, il s'agit du fondement pragmatique. On a parlé d'un refus solitaire de la pure analyse structurale au bénéfice d'une linguistique des opérations (cf. Dosse 1992, 60-74). A cet égard il y a éminemment un régime pragmatique dans la théorie de l'énonciation. Citons Benveniste à ce propos:

"De toute manière, un énoncé performatif n'a de réalité que s'il est authentifié comme acte" (PLG 1, 273).

Les deux fondateurs de la pragmatique, Morris et Austin, sont explicitement cités et discutés. Kerbrat-Orrechioni distingue la pragmatique énonciative de la pragmatique illocutoire ou théorie des actes du langage :

"En matière de pragmatique énonciative, Benveniste apparaît comme le véritable fondateur... Benveniste focalise son attention sur le seul événement". (1984, 48).

Autrement dit, Benveniste a cerné la dimension discursive du langage en révélant l'acte de discours. Mais pour ma part, je voudrais simplement signaler l'impact de Karl Bühler dans l'élaboration de la pragmatique continentale. En effet il a tenté d'élaborer une phénoménologie structurale de la deixis du langage, à savoir qu'il a défini déjà le discours comme champ monstratoire identifié à l'espace perceptif du locuteur (cf. Innis 1982).

En troisième et dernier lieu, on voit se disséminer l'option ou position phénoménologique dans la théorie énonciative. A ce propos nous avons besoin d'une étude plus approfondie et minutieuse, car il s'agit d'une jonction entre linguistique et phénoménologie qui se compénétrent. En tous cas Coquet a remarquablement démontré cette connivence entre l'oeuvre de Benveniste et la phénoménologie de Merleau-Ponty en reprenant quelques thèmes phénoménologiques dans l'oeuvre de Benveniste. Selon Coquet:

"C'est un des points où le linguiste est le plus en rupture avec la pensée dominante de son temps" (1992, 42).

En effet l'orientation phénoménologique se manifeste dans le primat du discours sur la langue. Benveniste affirme que :

"la langue se forme et se configure dans le discours" (PLG 1, 131).

Mais très curieusement dans le lexique de Benveniste on ne trouve ni la phénoménologie ni Husserl, ni Merleau-Ponty. Toutefois les notions phénoménologiques s'éparpillent un peu partout telles que: position, mouvement, centre de l'énonciation, instance, champ sémantique, ancrage, etc.

Mais cette hypothèse exige une problématique plus vaste, à savoir la connexion entre le structuralisme et la phénoménologie. Cette dernière a imprégné profondément la linguistique structurale. A titre d'exemple, H. Parret, dans une étude récente, a détecté une origine phénoménologique dans la théorie des cas de Hjelmslev. Au nom du réalisme et de l'efficacité le linguiste danois refuse le principe de binarisme : 
"Les termes d'une opposition sont exclusifs tandis que les positions dimensionnelles ne le sont pas. C'est dire que seule une conception dimentionnelle de la catégorie sera en état de penser la continuité et la gradualité. Hjelmslev, de ce point de vue, est de loin plus phénoménologique qu'algébriste" (Parret, 1995, 11).

Or bien avant, à la suite de Pos, Ricoeur a admis à cette orientation phénoménologique de la linguistique structurale. Il s'agit d'un structuralisme dynamique "intégrant le corps propre, les instances et leurs connexions" (Coquet 1993, 316). Il suffit de dire que Benveniste appartient à cette généalogie phénoménologique en laissant une maxime frappante à savoir que :"le langage est à vivre" (PLG Il, 217).

Cependant on remarque avec regret que Benveniste n'est pas allé jusqu'au bout; ni le concept de corps, ni celui de chair n'est présent chez lui contrairement à la philosophie du langage de Merleau-Ponty, chez qui c'est le corps lui-même qui est 'le noyau significatif" (Merleau-Ponty, 1945, 172). Le sens provient du corps. L'expression est le seul moyen technique pour matérialiser, former un signe susceptible de réaliser le sens vécu dans l'existence. Le corps n'investit pas le langage dans la seule finalité de communiquer, car la voix incarne le sens tout en extériorisant pour autrui dans le signe. En un mot le signe n'épuise pas le sens. Ainsi le phénoménologue refuse la présomption d'une dicibilité totale du langage. (cf. Andrieu, 1993). Cette insuffisance de l'expression accorde la place majeure à un primat de l'incarnation comme acte intérieur du langage.

3. Enfin essayons de dégager quelques limitations. D'abord sur le plan formel, on peut poser les faiblesses au niveau de l'intelligibilité. C'est ce que la critique sévère de Culioli a adopté. D'après lui, le texte de Benveniste se caractérise par "un surplus morphologique sans signification claire" $(1984,78)$. A titre d'exemple, Culioli relève ce défaut dans les textes sur la subjectivité :

"Ici encore, on rencontre une prolifération de désignations, et un concept manquant" (ibdi, 83).

Mais à mes yeux, les limites épistémologiques sont plus essentielles. La théorie de l'énonciation n'est pas suffisamment élaborée, elle ne peut traiter que quelques propriétés spécifiques du discours. Je m'appuie ici sur la critique de Parret :

"tout aspect de la réalisation, de l'actualisation de fragments linguistiques n'est pas dans le discours, puisque la suite infinie des événements de parole n'est pas repérable comme une suite d'instances $d u$ discours. C'est ainsi que l'on peut se méfier du projet même de la 'linguistique énonciative' de Benveniste, bien que ce linguiste..." (1987, 84).

$\mathrm{Du}$ fait de l'écart entre l'objet théorique et le réel phénoménologique le projet de la théorie de l'énonciation qui envisage la substance de l'événement 
n'est pas fondé en termes épistémologiques. De surcroît elle n'est pas axiomatisée excepté le domaine précis de la deixis discursive. Enfin on constate que la théorie des modalités est presque absente dans l'appareil de l'énonciation. Bref, malgré l'intérêt heuristique pour l'analyse discursive il y a peu d'impact de théorisation dans le domaine global de la modélisation sémiotico-sémantique.

\section{Bibliographie}

B. ANDRIEU, «Le langage entre chair $<$ Leib $>$ et corps $<$ Körper» $>$, in :Recherches sur la philosophie et le langage N.15, 1993.

M.ARRIVÉ, «Y-a-t-il en glossématique une théorie de l'énonciation ?», HEL VIII-2, 1986.

E. BENVENISTE, Problèmes de linguistique générale I et II, Paris, Gallimard, 1966, 1974.

J-CI. COQUET, «Linguistique et sémiologie», Actes sémiotiques-Docunents IX : 88, 1987, 5-20.

J.-CI. COQUET, «Réalité et principe d'immanence», Langages 103, 191, 1.

J.-CI. COQUET, «Note sur Benveniste et sur la phénoménologie», LINX 26, 1992.

J.-CI. COQUET, «L'objet dans l'optique d'une phénoménologie linguistique», Revue Européenne d'études Sémiotiques Vol.5-1,2, 1993, 307-318.

A. CUliOLI, «Théorie du langage et théorie des langues», in :E.Benveniste Aujourd'hui tome 1 éd. Guy Serbat, 1984, 77-85.

S. DELESALLE (sous la direction), Histoire des conceptions de l'énonciation, HEL VIII-2, 1986.

F. DossE, Histoire du structuralisme, tome 2, Paris, Editions la découverte, 1992.

0. DUCROT, «Structuralisme, énonciation et sémantique», dans Poétique, 1978, 33, 107-128.

0, DUCROT, «Enonciation», dans le Supplément de Encyclopédia Universalis, 1980.

0. DuCROT, Les mots du discours, Paris, Ed. de Minuit 1980.

M. FOUCAUlt L'archéologie du savoir, Paris, Ed. de Minuit, 1980.

A.J. GReIMAS et J. COURTÉs, Sémiotique. Dictionnaire raisonné de la théorie du langage, t. 1. Paris, Hachette, 1979 et t.II, Paris, Hachette, 1985.

L. HJELMSLEV, Le langage, Paris, Ed. de Minuit, 1966.

R.E. INNIS, Karl Bühler Semiotic Foundations of Language Theory, New York, Plenum Press, 1982.

F. JACQUES, Différence et Subjectivité, Paris, Aubier-Montaigne, 1982.

F. JACQUES, «La mise en communauté de l'énonciation», Langages, 1983, 70, 47-71.

F. JACQUES, L'espace logique de l'interlocution, Paris, PUF, 1985.

C. KERBRAT-ORECCHIONI, «E. Benveniste et la théorisation: la pragmatique du langage», in : E. Benveniste Aujourd'hui tome 1 éd. Guy Serbat, 1984, 45-55. 


\section{Sungdo Kim}

D. MaIngUeneaU, Genèse du discours, Bruxelles, Mardaga, 1984.

M. MERLEAU-PONTY, Phénoménologie de la perception, Paris, Gallimard, 194r..

CI. NORMAND, «Le sujet dans la langue», Langages 77, 1985, 7-19.

CI. NORMAND, «Constitution de la sémiologie chez Benveniste», HEL 11, 1989.

H. PARRET, «L'énonciation en tant que déictisation et modalisation», dans Langages (La mise en discours), 1983, 70, 83-98.

H. PARRET, Prolégomènes à la théorie de l'énonciation De Husserl à la pragmatique, Berne, Peter Lang, 1987.

H. PARRET, «Préhistoire, structure et actualité de la théorie des cas», Actes sémiotques, 1995.

P. RICOEUR, «L'Homme et le langage», Tendances principales de la recherche dans les sciences sociales et humaines 2,2. Paris-la Haye : Mouton-Unesco. 